\title{
The Lund Model at Nonzero Impact Parameter*
}

\author{
Romuald A. Janik ${ }^{a}$, Robi Peschanski ${ }^{b \dagger}$ \\ ${ }^{a}$ Jagellonian University, \\ Reymonta 4, 30-059 Krakow, Poland. \\ ${ }^{b} \mathrm{CEA} / \mathrm{DSM} / \mathrm{SPhT}$ Saclay \\ Unité de Recherche associée au CNRS \\ CEA-Saclay, F-91191 Gif/Yvette Cedex, France.
}

December 24, 2018

\begin{abstract}
We extend the formulation of the longitudinal $1+1$ dimensional Lund model to nonzero impact parameter using the minimal area assumption. Complete formulae for the string breaking probability and the momenta of the produced mesons are derived using the string worldsheet Minkowskian helicoid geometry. For strings stretched into the transverse dimension, we find probability distribution with slope linear in $m_{T}$ similar to the statistical models but without any thermalization assumptions.
\end{abstract}

\section{Introduction}

One phenomenological ingredient of models for hadronic reactions is the description of the hadronization process when the (hard) partons produced at

${ }^{*}$ Dedicated to the memory of Bo Andersson.

${ }^{\dagger}$ e-mail: ufrjanik@if.uj.edu.pl, pesch@spht.saclay.cea.fr 
the interaction point get dressed to form the observed hadronic particles which are registered in the detector. This is a theoretically difficult problem due to its nonperturbative character, however phenomenological models are quite successful in the description of data. Models which describe this behaviour are essential for the comparision of QCD predictions for partonic processes with the experimental data.

One of the most successful hadronization models is the Lund model [1, 2 based on the effective string picture of QCD at strong coupling. The conventional Lund model ${ }^{1}$ gives a description of the break-up of a colour string into produced hadronic states. It is directly applicable in the context of processes where the initial $q \bar{q}$ pair is produced at a single point, the partons then fly away from each other, a colour string is stretched between them, which eventually breaks up producing hadrons. A fundamental property of the confining strings is the so-called area law [4, from which the probability formula in the Lund model may be advocated.

The model is basically $1+1$ dimensional, transverse momenta of the generated mesons are taken into account by substituting the masses $m^{2}$ by $m_{T}^{2}=m^{2}+k_{\perp}^{2}$. In particular the transverse momenta are considered to be in principle randomly distributed, decorrelated along the chain of mesonic emissions and also uncorrelated with rapidity.

The aim of this paper is to show that the whole $1+1$ dimensional setup of the Lund fragmentation model based on the area law can be generalized to the $3+1$ case when the initial quark and antiquark are also separated by a transverse distance $L$ (impact parameter). These types of colour string configurations may occur between coloured remnants of a projectile and of the target. They could also appear locally in the hadronization of a partonic cascade between neighbouring partons at some transverse distance.

Our results are the following. We give the full mathematical derivation of the probability distribution function for the production of mesons with their 4-momenta ((11) and formulae (22)-(24)). This is based on the Minkowskian helicoid geometry which is the relevant string worldsheet for nonzero impact parameter $L$. The results depend on the key parameter $p=\chi / L$, where $\chi$ is the rapidity interval. For large $p$ we recover the Lund model probability distribution, while for small $p$ new features appear. We obtain a linear slope in $m_{T}$ and not $m_{T}^{2}$ as is usually assumed in effective string models. This

\footnotetext{
${ }^{1}$ By the 'conventional' Lund model we mean the original $1+1$ dimensional formulation not containing the extension to gluonic strings $[3$.
} 
derivation based just on classical considerations has to be contrasted with similar predictions based on statistical model assumptions.

The plan of this paper is as follows. We will first review the standard $1+1$ Lund model along the lines of [5]. Then in section 3 we will introduce the Minkowskian helicoid minimal surface which is crucial for the construction of the generalization to nonzero impact parameter. In section 4 we will show how the basic formulae of the Lund model get modified in the new setting. In section 5 we will analyze the limiting cases of small and large $p$. In section 6 we close the paper with considerations of phenomenological interest.

\section{$2 \quad 1+1$ dimensional Lund model}

Suppose that a highly energetic $q \bar{q}$ pair is produced at the point $x^{\mu}=0$. These particles move along straight lines

$$
x_{0}=\frac{v_{0}}{2} t_{0} \quad \bar{x}_{0}=-\frac{v_{0}}{2} \bar{t}_{0}
$$

with relative rapidity

$$
\chi=\frac{1}{2} \log \frac{1+v_{0}}{1-v_{0}} .
$$

According to the effective string picture of QCD, a colour string is formed between them. Since by assumption we are in the confining regime, the string lies in the most energetically favourable configuration i.e. it forms the minimal surface joining the two lines (see fig. 1a). This surface is of course flat in this case. This follows from the Nambu-Goto action for a confining string worldsheet $S_{N G}=\kappa \cdot$ Area.

The process of hadronization is described by the break up of this string (see fig. 1b) forming a pair of massless quarks, each of which moves along the light cone directions and which get converted into hadrons (at the upper tips in fig. 1b). The probability of such a breakup configuration is given by the area law:

$$
\text { Probability } \sim \exp (-b \cdot \text { Area })
$$

where Area is the area spanned by the string and $b$ is a phenomenological parameter which is related to the break-up probability of the string per unit area [2].

The area can be calculated as a sum of the areas of the diamond-shaped sectors (like the one in fig. 1b between the dotted lines). Denoting the 

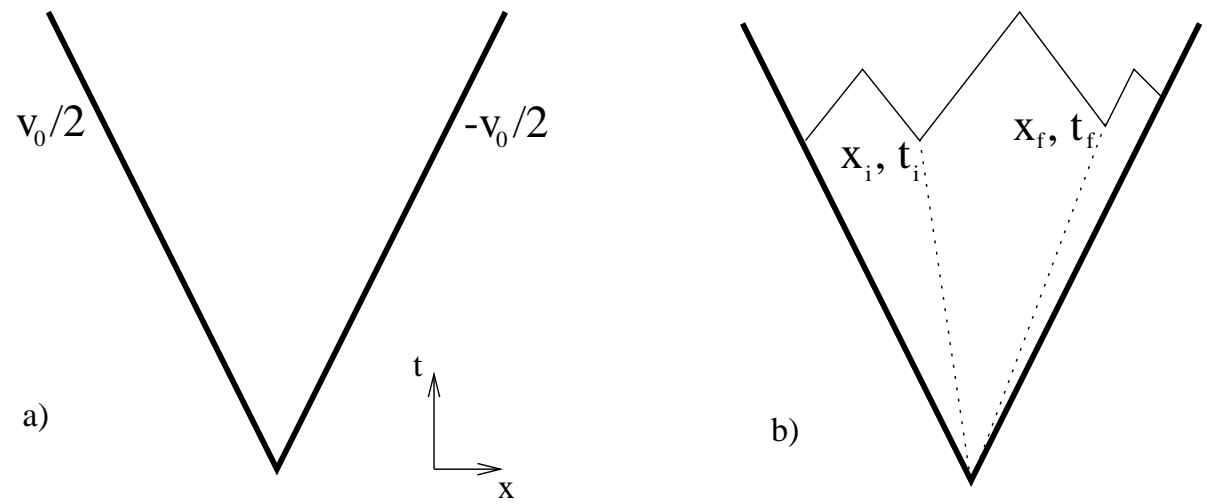

Figure 1: Configuration with zero impact parameter. a) Initial $q \bar{q}$ configuration; b) a sample configuration of the confining string with break-up points (the dotted lines divide the string area into diamond-like sectors - see text).

coordinates of the adjacent points of breakup by $\left(t_{i}, x_{i}\right)$ and $\left(t_{f}, x_{f}\right)$ one obtains the formula

$$
\text { Area }_{i f}=\frac{1}{4}\left(2 x_{-i} x_{+f}-x_{+f} x_{-f}-x_{+i} x_{-i}\right)
$$

where

$$
x_{ \pm}=t \pm x .
$$

At this stage the essential probabilistic set-up of the Lund model is complete.

The second ingredient in the Lund model is a prescription how to associate the produced physical mesons to the string break-up configuration characterized by the break-up points $\left(t_{k}, x_{k}\right)$.

Let the string worldsheet be parameterized by the coordinates $\tau$ and $\sigma$. Then the Nambu-Goto lagrangian

$$
\mathcal{L}=\kappa \sqrt{-\left(h_{\tau \tau} h_{\sigma \sigma}-h_{\tau \sigma}^{2}\right)}
$$

is expressed in terms of the induced metric

$$
h_{a b}=\partial_{a} x^{\mu} \partial_{b} x_{\mu} .
$$

The 4-momentum of the produced meson formed between the adjacent points of breakup $\left(t_{i}, x_{i}\right)$ and $\left(t_{f}, x_{f}\right)$ follows from the formula for the momentum of a (classical) string stretched between these points [6]:

$$
P^{\mu}=\int_{C} \frac{\partial \mathcal{L}}{\partial \dot{x}^{\mu}} d \sigma-\frac{\partial \mathcal{L}}{\partial x^{\prime \mu}} d \tau
$$


where $C$ is (any) curve joining the breakup points. For flat $\left(h_{a b}=\eta_{a b}\right)$ or more generally conformally flat coordinates $\left(h_{a b}=e^{\Phi(\sigma, \tau)} \eta_{a b}\right)$ this simplifies to

$$
P^{\mu}=\kappa \int_{C}\left(\dot{x}^{\mu} d \sigma+x^{\prime \mu} d \tau\right) .
$$

Applying (8) to the flat minimal surface shown in fig 1b yields

$$
\begin{aligned}
& P^{0}=\kappa\left(x_{f}-x_{i}\right) \\
& P^{1}=\kappa\left(t_{f}-t_{i}\right) \\
& P_{\perp}=0 .
\end{aligned}
$$

The final ingredient, which is not derived from the model, is the mass shell condition for the produced mesons. Thus $\delta\left(P^{2}-m^{2}\right)$ is put in where $m^{2}$ are the physical meson masses.

The resulting probability distribution function [1] for the production of mesons with momenta $\left\{p_{j}\right\}$ is thus:

$$
d P_{n}\left(\left\{p_{j}\right\}, P_{t o t}\right) \propto \prod_{j=1}^{n} d^{2} p_{j} \delta\left(p_{j}^{2}-m_{j}^{2}\right) \delta\left(\sum_{j} p_{j}-P_{t o t}\right) \exp (-b \cdot \text { Area })
$$

where

$$
\text { Area } \equiv \sum \text { Area }_{i f}
$$

is the total area of the string worldsheet (see fig. 1b).

Note that all the above steps and formulae, apart from the mass-shell condition, follow just from the geometry of the flat surface between the initial lines, which in turn is unambigously determined as the minimal surface between the trajectories of the initial energetic $q \bar{q}$ pair which generates the initial colour string. The point that we would like to emphasize here is that it is possible to use this property to generalize the model to configurations with nonzero impact parameter.

\section{The minimal surface geometry: the Mink- owskian helicoid}

When the string is formed between a quark and antiquark, moving with relative rapidity $\chi$, and separated by a transverse distance $L$, the string worldsheet will stretch along the minimal surface formed by the two lines. 

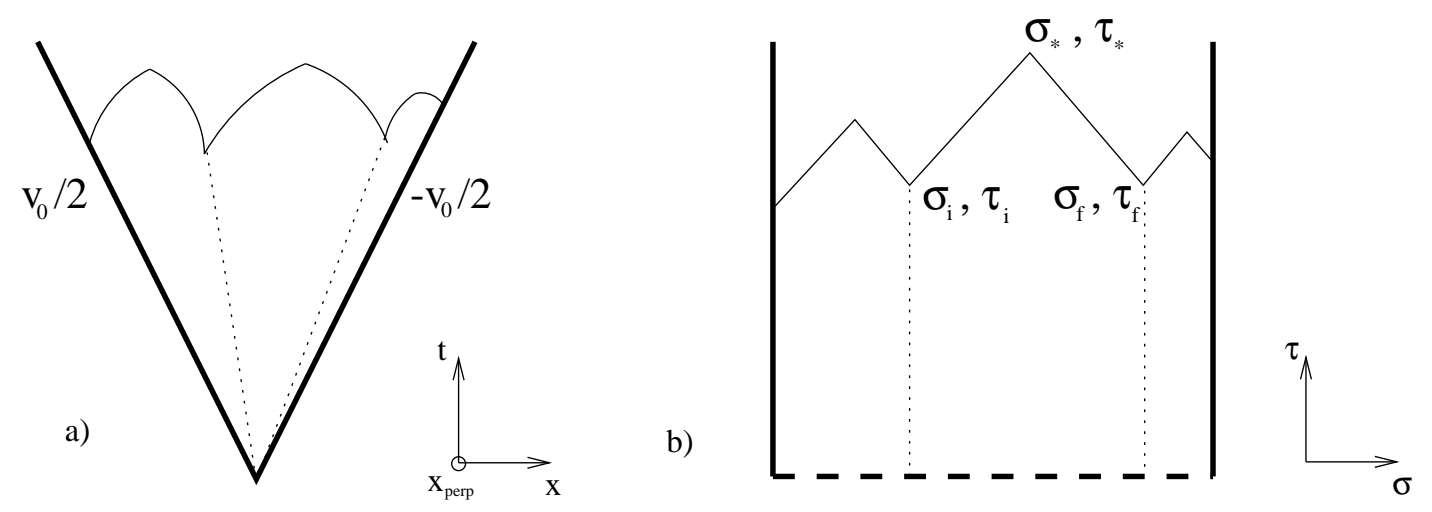

Figure 2: A string breaking on the helicoid. a) picture projected onto the longitudinal plane (to be compared with fig. 1b); b) the same configuration in the $\tau-\sigma$ variables.

An Euclidean version of this surface has been used in an approach to study high energy scattering using the AdS/CFT correspondence [7].

The minimal surface here ${ }^{2}$ is a (Minkowskian) M-helicoid which can be parametrized by

$$
\begin{aligned}
t & =\frac{1}{p} \sinh p \tau \cosh p \sigma \\
x & =\frac{1}{p} \sinh p \tau \sinh p \sigma \\
x_{\perp} & =\sigma
\end{aligned}
$$

where $p=\chi / L, \sigma=-L / 2 \ldots L / 2$ and $\tau$ starting $^{3}$ at 0 . The string at $t=0$, when the particles are separated by a transverse distance equal to the impact parameter, is stretched along a straight line.

The geometry of the helicoid is encoded in the induced conformally flat metric:

$$
h_{a b}=\left(\cosh ^{2} p \tau\right) \cdot \eta_{a b} .
$$

\footnotetext{
${ }^{2}$ The assumption that the string at $t=0$ is a straight segment in impact parameter selects the helicoid minimal surface. If the initial string would be in some excited configuration the analysis would possibly lead to different solutions. Note that an a priori different transverse configuration, going beyond the original Lund model, has been studied in 8 with a numerical treatment based on the ARIADNE 2 program 9 .

${ }^{3}$ Our formalism can be easily extended to other initial conditions for $\tau$.
} 
Hence

$$
d s^{2}=\cosh ^{2} p \tau \cdot\left(d \tau^{2}-d \sigma^{2}\right) .
$$

Since the produced quarks are assumed to be massless, they follow light-like trajectories $\left(d s^{2}=0\right)$ on the helicoid which are just straight lines at an angle of $\pm 45^{\circ}$ in the $\tau-\sigma$ variables. The area element is

$$
d \mathcal{A}=\sqrt{-\operatorname{det} h}=\cosh ^{2} p \tau d \tau d \sigma .
$$

It is also convenient to look at the helicoid projected onto the longitudinal $t-x$ plane. Then one can show that

$$
\frac{x}{t}=\tanh p \sigma \quad \quad p \sigma=\frac{1}{2} \log \frac{x_{+}}{x_{-}} .
$$

So the lines of constant $\sigma$ correspond to fixed longitudinal velocities ${ }^{4} v$.

The $\tau$ coordinate can also be related to the longitudinal coordinates through

$$
\sinh p \tau=p \sqrt{x_{+} x_{-}} .
$$

As we saw in the previous section all ingredients of the Lund model can be formulated as purely geometric constructions based on the minimal surface which represents the colour string. We thus have to redo the calculations of section 2 substituting the helicoid for the original flat surface.

\section{The Lund model at nonzero impact param- eter}

We will now derive the formulae for the break-up probability and produced momenta for the nonzero impact parameter case which are enough to define the generalization of the full probability distribution function (11). The only thing that changes is the underlying geometry which is no longer flat but is given by the Minkowskian helicoid. It is convenient to label the breakup points by the longitudinal coordinates $(t, x)$ (i.e. to look at the helicoid projected onto the $t-x$ plane) and subdivide the area into same sectors as before (the boundaries being lines of constant slope $x / t=v=$ const, i.e.

\footnotetext{
${ }^{4}$ This is also the definition of the boundaries of the sectors in fig. $1 \mathrm{~b}$ in the original zero impact parameter model.
} 
constant $\sigma$ ). In fig. 2a we show the breakup in the $t-x$ plane while in fig. $2 \mathrm{~b}$ we depict the same process 5 . .

We calculate first the area of the sector defined by the breaking up points $\left(\sigma_{i}, \tau_{i}\right)$ and $\left(\sigma_{f}, \tau_{f}\right)$. The coordinates of the tip of the sector are:

$$
\sigma_{*}=\frac{\tau_{f}-\tau_{i}+\sigma_{f}+\sigma_{i}}{2} \quad \tau_{*}=\frac{\tau_{f}+\tau_{i}+\sigma_{f}-\sigma_{i}}{2} .
$$

The area is then given by

$$
\text { Area }_{i f}=\int_{\sigma_{i}}^{\sigma_{*}} d \sigma \int_{0}^{\tau_{i}+\sigma-\sigma_{i}} \cosh ^{2} p \tau d \tau+\int_{\sigma_{*}}^{\sigma_{f}} d \sigma \int_{0}^{\tau_{f}-\sigma+\sigma_{f}} \cosh ^{2} p \tau d \tau
$$

Using the integral

$$
\int_{0}^{s} \cosh ^{2} p \tau d \tau=\frac{s}{2}+\frac{\sinh (2 p s)}{4 p}
$$

the $A r e a_{i f}$ can be calculated in closed form:

$$
\begin{aligned}
\text { Area }_{i f} & =\frac{1}{8}\left(\left(\sigma_{f}-\sigma_{i}\right)^{2}-\left(\tau_{f}-\tau_{i}\right)^{2}+2\left(\sigma_{f}-\sigma_{i}\right)\left(\tau_{f}+\tau_{i}\right)\right)+ \\
& +\frac{1}{8 p^{2}}\left(2 \cosh \left(p\left(\sigma_{f}-\sigma_{i}+\tau_{f}+\tau_{i}\right)\right)-\cosh \left(2 p \tau_{f}\right)-\cosh \left(2 p \tau_{i}\right)\right)
\end{aligned}
$$

We now have to compute the momentum carried by the string between the breakup points. This is identified with the 4-momentum of the produced meson. Using formula (9) we get

$$
\begin{aligned}
P^{0} & =\frac{\kappa}{p}\left(\cosh p \tau_{f} \sinh p \sigma_{f}-\cosh p \tau_{i} \sinh p \sigma_{i}\right) \\
P^{1} & =\frac{\kappa}{p}\left(\cosh p \tau_{f} \cosh p \sigma_{f}-\cosh p \tau_{i} \cosh p \sigma_{i}\right) \\
P_{\perp} & =\kappa\left(\tau_{f}-\tau_{i}\right) .
\end{aligned}
$$

\footnotetext{
${ }^{5}$ We should emphasize that in contast to the break-up points, which have a definite physical meaning, the subdivision of the string worldsheet into sectors is to a large extent arbitrary. Our choice here was to subdivide the area in a way similar to the flat string case in order to facilitate the comparison with the $1+1$ dimensional Lund model. Of course the final probabilistic formulas contain only the sum of all sectors which is uniquely defined in the $\tau-\sigma$ variables.
} 
It is convenient, also for comparison with the zero impact parameter case to rewrite this in terms of longitudinal coordinates

$$
\begin{aligned}
P^{0} & =\kappa\left(\sqrt{1+\frac{1}{p^{2} x_{+f} x_{-f}}} x_{f}-\sqrt{1+\frac{1}{p^{2} x_{+i} x_{-i}}} x_{i}\right) \\
P^{1} & =\kappa\left(\sqrt{1+\frac{1}{p^{2} x_{+f} x_{-f}}} t_{f}-\sqrt{1+\frac{1}{p^{2} x_{+i} x_{-i}}} t_{i}\right) \\
P_{\perp} & =\frac{\kappa}{p}\left(\log \left[\sqrt{1+p^{2} x_{+f} x_{-f}}+p \sqrt{x_{+f} x_{-f}}\right]-(f \rightarrow i)\right) .
\end{aligned}
$$

Formulae (22), (23) and equivalently (24) when inserted into (11) completely define our model and can be used as a basis of a Monte-Carlo simulation.

\section{The limits of small and large aspect ratio $p$}

The key parameter which characterizes the surface geometry is $p=\chi / L$. The scale of the momenta of the produced mesons is set by the string tension $\kappa$.

\section{Small impact parameter}

Let us first verify that when $p \rightarrow \infty$ (large rapidity or zero impact parameter limit) we recover the formulae of the conventional Lund model. Indeed it is clear from (24) that the momenta of the particle produced go over to the conventional Lund expressions (note that $P_{\perp} \sim(\log p) / p \rightarrow 0$ ). In fact we have to impose the condition that $x_{ \pm}$of the break-up points are kept fixed in the limit. From (17) this means that $\sigma \rightarrow 0$ with $p \sigma$ fixed.

The Area of each sector (22) also reduces to the Lund expression (41). To see this we note that for large $p$ (and keeping the longitudinal coordinates of the break up points fixed) the polynomial terms in (22) vanish, the only contribution comes form the hyperbolic cosines. This can be easily evaluated using $e^{p \sigma}=\sqrt{x_{+} / x_{-}}$and $e^{p \tau} \sim 2 p \sqrt{x_{+} x_{-}}$(for $p \rightarrow \infty$ ). The result is the Lund expression (41).

Since we obtained the expression (44) where there is no explicit remaining $p$ dependence, our assumption that the break up longitudinal coordinates do not scale with $p$ but remain of order 1 is self-consistent. In this way we 
verified that the conventional Lund model is a smooth limit of the nonzero impact parameter generalization.

\section{Large impact parameter}

It is especially interesting to study which new phenomenae may arise in comparision to the original model, and whether these are phenomenologically important. To answer this question in full one has to perform a Monte Carlo simulation. Here we will just consider the limit of large impact parameter (small $p$ limit) where one can expect the largest deviations from the original Lund model.

Before we make the analysis let us note that the string worldsheet in the $p \rightarrow 0$ limit is a flat string stretched in the transverse plane which is still unbroken at $t=0$. Then it will fragment into mesons which will have nonzero transverse momenta even at the classical level.

Let us check how does the area scale when $p \rightarrow 0$. If we where to keep the longitudinal coordinates fixed and of order 1 we would arrive at a singular area $^{6}$ ('action') for $p \rightarrow 0$. Hence the above assumption in this case is unjustified. The correct choice is in fact that the coordinates $(\sigma, \tau)$ remain of order 1. This is natural physically as the transverse mass in the small $p$ limit stays finite and becomes $m_{T}=\kappa \Delta \sigma \equiv \kappa\left(\sigma_{f}-\sigma_{i}\right)$. The $\tau$ coordinate is also directly linked to the transverse momentum through $\Delta \tau=P_{\perp} / \kappa$.

Considering now $\sigma$ and $\tau$ fixed and starting with the area formula (22) one gets for $p \rightarrow 0$ :

$$
\text { Area }_{i f} \rightarrow \frac{1}{4}\left[\Delta^{2} \sigma-\Delta^{2} \tau\right]+\tau^{+} \Delta \sigma \sim \frac{m^{2}}{4 \kappa^{2}}+\tau^{+} \frac{m_{T}}{\kappa},
$$

where

$$
\Delta \sigma \equiv \sigma_{f}-\sigma_{i} ; \Delta \tau \equiv \tau_{f}-\tau_{i} ; \tau^{+} \equiv \frac{\tau_{f}+\tau_{i}}{2} .
$$

Therefore the break-up probability for the formation of a meson takes the

\footnotetext{
${ }^{6}$ One gets then

$$
\text { Area }_{x_{ \pm}}=\frac{1}{8 p^{2}}\left[\frac{x_{-i} x_{+_{f}}+x_{-f} x_{+i}}{\sqrt{x_{-i} x_{+i}} \sqrt{x_{-f} x_{+f}}}-2\right]+\frac{1}{32 p^{2}}\left(\log \frac{x_{+f}}{x_{-f}}-\log \frac{x_{+i}}{x_{-i}}\right)^{2} .
$$
}


following simple form

$$
\text { Probability } \propto \exp \left(-\frac{b m^{2}}{4 \kappa^{2}}\right) \cdot \exp \left(-\frac{b m_{T}}{\kappa} \tau^{+}\right),
$$

where $\tau^{+}$can be related to the mean time value of two adjacent break-up points in the limit $p \rightarrow 0$. Indeed, one has

$$
\sqrt{t_{i}^{2}-x_{i}^{2}}+\sqrt{t_{f}^{2}-x_{f}^{2}}=\frac{1}{p} \sinh p \tau_{i}+\frac{1}{p} \sinh p \tau_{f} \rightarrow \tau_{i}+\tau_{f} \text { when } p \rightarrow 0 .
$$

\section{Phenomenological considerations}

Formula (27) has quite intriguing properties. Let us assume that the value of $\tau^{+}$fluctuates around some average value $\langle\tau\rangle$. We emphasize that this assumption should be checked in a complete Monte-Carlo simulation. Then the distribution of produced particles is predicted to follow a simple formula

$$
\exp \left(-\frac{b m^{2}}{4 \kappa^{2}}\right) \cdot \exp \left(-\frac{b m_{T}}{\kappa}\langle\tau\rangle\right)
$$

with a slope linear in $m_{T}$. This is quite unusual in string based models where generically a behaviour quadratic in $m_{T}^{2}$ appears ${ }^{7}$ [10 in relation with the Schwinger mechanism 11. Here the slope linear $m_{T}$ arises due to subtle cancellations between quadratic terms in the helicoid area and thus appears directly at the classical level.

It is interesting to compare these properties with those of the generic statistical model [12] which successfully describes both the slope in $m_{T}$ and the abundances of different particle species in high energy reactions [13. In this model the creation of particles is supposed to come from thermalized clusters of limited center of mass energy. Our model, at small $p$, leads to similar conclusions but without invoking the thermalization assumption. Indeed we have checked that the slope in $m_{T}$ and the average relative distribution of the multiplicities of different species can be qualitatively described by a simple formula of the form (29). In order to go further and to determine the parameters like $\langle\tau\rangle$ one needs to perform a complete Monte-Carlo simulation which goes beyond the scope of this paper.

\footnotetext{
${ }^{7}$ In ref. 10 it was suggested that the phenomenologically preferred behaviour linear in $m_{T}$ can be obtained by considering gaussian fluctuations of the string tension.
} 
Let us finally note another possible implication of the string breaking model based on the helicoid geometry. Since the helicoid is extended in the transverse direction, transverse momenta get produced at the classical level on the same footing as longitudinal ones. This opens up an exciting possibility that correlations between $p_{t}$ and rapidity could be naturally incorporated in the model. We leave a detailed investigation of these issues which should be based on a Monte Carlo simulation for future work ${ }^{8}$.

Acknowledgments. A discussion with our deeply missing friend Bo Andersson at the Weimar Conference (2001) is to be acknowledged. We thank Gosta Gustafson, Gunnar Ingelman and Torbjorn Sjostrand for discussions. RJ would like to thank SPhT Saclay for hospitality when this work was completed. RJ was partially supported by KBN grant 2P03B09622 (2002-2004).

\section{References}

[1] B. Andersson, G. Gustafson, G. Ingelman and T. Sjostrand, Phys. Rept. 97 (1983) 31; B. Andersson, G. Gustafson and B. Soderberg, Z. Phys. C 20 (1983) 317;

[2] X. Artru and G. Mennessier, Nucl. Phys. B 70 (1974) 93; X. Artru, Phys. Rept. 97 (1983) 147.

[3] B. Andersson, S. Mohanty and F. Soderberg, arXiv:hep-ph/0212122.

[4] K. G. Wilson, Phys. Rev. D 10 (1974) 2445.

[5] B. Andersson and F. Soderberg, Eur. Phys. J. C 16 (2000) 303 arXiv:hep-ph/9910374.

[6] See e.g. formula (3.31) in B. M. Barbashov and V. V. Nesterenko, "Introduction To The Relativistic String Theory," Singapore, World Scientific (1990).

[7] R. A. Janik and R. Peschanski, Nucl. Phys. B 586 (2000) 163 arXiv:hep-th/0003059; R. A. Janik, Phys. Lett. B 500 (2001) 118 arXiv:hep-th/0010069; R. A. Janik and R. Peschanski, Nucl. Phys. B 625 (2002) 279 arXiv:hep-th/0110024.

\footnotetext{
${ }^{8}$ We thank the referee for suggesting this problem.
} 
[8] B. Andersson, G. Gustafson, L. Lönnblad and U. Petterson, Z. Phys. C 43 (1989) 625.

[9] U. Pettersson: LU-TP-88-5; L. Lonnblad, U. Pettersson LU-TP-88-15.

[10] A. Bialas, Phys. Lett. B 466 (1999) 301 arXiv:hep-ph/9909417.

[11] J. S. Schwinger, Phys. Rev. 128 (1962) 2425.

[12] R. Hagedorn, Nuovo Cim. Suppl. 3 (1965) 147.

[13] F. Becattini, Z. Phys. C 69 (1996) 485; F. Becattini and G. Passaleva, in high energy collisions," Eur. Phys. J. C 23 (2002) 551 arXiv:hep-ph/0110312. 